\title{
Renormalization Group Analysis of Some Dynamical Systems with Noise
}

\author{
P. Collet ${ }^{1}$ and A. Lesne ${ }^{1}$
}

Received March 6, 1989; revision received July 8, 1989

\begin{abstract}
We formulate a renormalization group analysis for the study of the accumulation of period doubling in the presence of noise. The main tool is a renormalization of the time evolution of the noise. The critical indices depend on the nature of the noise, but are given by thermodynamic quantities describing the large deviations of the Lyapunov exponent of the linearized random renormalization.
\end{abstract}

KEY WORDS: Period doubling; renormalization; Lyapunov exponents; large deviations.

\section{INTRODUCTION}

The influence of noise on the accumulation of period doubling bifurcations was studied several years ago by several authors. ${ }^{(5,8,9,19,21)}$ They discovered a new universal number with a value of about $6.6192 \ldots$. One possible way of describing this number is as follows. On the bifurcation diagram of the so-called standard one-parameter family of mappings of the interval $[-1,1]$ given by

$$
x \rightarrow 1-\mu x^{2}
$$

for $\mu$ in the interval $[0,2]$, one can observe consecutive windows of values of the parameter $\mu$ corresponding to stable periodic orbits of period 1 , then 2 , then 4,8 , etc. In the presence of noise, the smallest windows are washed out and the boundaries of the remaining ones consist of fuzzy bifurcations. The universal number is associated with the following question: by how much should one decrease the amplitude of the noise to observe one more

\footnotetext{
${ }^{1}$ Centre de Physique Théorique de l'École Polytechnique (Laboratoire UPRA 14 du CNRS), F91128 Palaiseau, France.
} 
level of windows? Asymptotically, the answer is the above universal number. This result was usually obtained for an additive noise composed of independent identically distributed Gaussian random variables. Similar results were observed for an additive noise coming from an irrational rotation on the circle in refs. 1 and 2 . In particular, a seemingly nearby value for the universal number appeared (except for some special irrational numbers). This nice observation is the basis of our method to analyze the problem. As we shall see below, the quasiperiodic case turns out to be simpler than the random case. Both cases have been analyzed in the context of a linear renormalization group analysis and a similar renormalization equation was found. We shall present here a more general approach which allows us to analyze a much broader class of noises and which is suited to a nonlinear analysis. Our approach differs from the old one in several ways. In particular, we shall not renormalize the noise, but the time evolution of the noise. As we shall see, this is a more natural renormalization which works for all noises. We shall explain how the previous analysis emerges from this more general approach.

We shall describe first the complete renormalization transformation in the presence of bounded noise and derive the linear renormalization mapping. The case of unbounded noise can also be treated; however, one should deal with a problem of large deviations when the mapping does not leave the interval $[-1,1]$ invariant. We shall then study a special problem of multiplication of random operators which turns out to be the building block of all the linear renormalization maps. However, due to large-deviation effects in Lyapunov exponents, one may observe different universal numbers for different noises, as we shall see below. In the cases mentioned above, the universal numbers turn out to be very close; however, one can show that they are different in $\varepsilon$ expansion.

The outcome of the analysis is the existence of a positive universal exponent due to the presence of the noise (this can be proven rigorously in $\varepsilon$ expansion). This exponent leads to a crossover phenomenon (which our theory predicts to be of infinite dimension). If one adds a small noise to the mapping, it is easy to show that the Cantor structure will remain up to a certain scale for the (nonautonomous) random iteration. The big gaps in the Cantor set which are associated with the periodic orbits of small period will remain. If the renormalization amplifies the noise, then even the large gaps of the Cantor set will eventually be destroyed. A rough estimate is that they are destroyed when the amplitude of the noise is of the order of their size. This argument gives a qualitative explanation of the numerical results described at the beginning of this section. A rigorous argument should be based on considerations involving support properties of invariant measure. We refer to ref. 23 for an analysis based on this idea. 
In Section 2, we describe a general formalism for renormalization in the presence of noise, which is a generalization of the usual one. We also derive a general formula for the linearized problem. In Section 3, we treat the particular case of a quasiperiodic noise. As we shall see, the universal number depends on the regularity of the mapping with respect to the noise. In order to give results which apply to more general maps than simple trigonometric polynomials, one has to study the large deviations of a Lyapunov exponent. We develop this technique in Appendix A. In Appendix B, we show that the hypotheses of Appendix A are satisfied in $\varepsilon$ expansion. We also mention that under the same hypothesis our method applies in a more general context and provides a large-deviation-theorem (" $f(\alpha)$ " result) for the largest Lyapunov exponent (see ref. 4 for a onedimensional analogue). In Appendix $\mathrm{C}$, we derive some results about the nonlinear analysis.

\section{RANDOM ITERATIONS AND RENORMALIZATION}

We shall denote by $X$ the phase space of our system, and assume that it is a compact topological space. We shall be mostly dealing with the case $X=[-1,1]$, although it is easier at this point to adopt a more abstract point of view. Several formalisms can be used to describe random iterations on $X$. For our purposes, the quasiproduct formalism seems more convenient, and we now briefly recall this approach. We assume that a measurable space $Y$ (the phase space of the noise) is given with a probability measure $v$. The time evolution of the noise is given by an invertible measurable map $g$ (with measurable inverse) of $Y$ preserving the measure $v$. For example, in the case of independent random variables, $Y$ is an infinite product space and the time evolution is the shift, while for a quasiperiodic noise, $Y$ is the circle and the time evolution is a rotation (which may be rational or not). We define a random iteration map to be a measurable map $F$ from $X \times Y$ to $X$. We shall of course assume later on some regularity for $F$. We can now consider the map $\widetilde{F}$ of $X \times Y$ which is given by

$$
\tilde{F}:\left(\begin{array}{l}
x \\
y
\end{array}\right) \rightarrow\left(\begin{array}{c}
F(x, y) \\
g(y)
\end{array}\right)
$$

The $n^{\text {th }}$ (random) iterate of $F$, denoted by $F^{[n]}$, is the projection on $X$ of the $n^{\text {th }}$ iterate $\widetilde{F}^{(n)}=\widetilde{F} \circ \widetilde{F} \circ \ldots \circ \widetilde{F}\left(n\right.$ times) of $\widetilde{F}$. In other words, $F^{[n]}$ satisfies the following recursion relation:

$$
F^{[n]}(x, y)=F\left(F^{[n-1]}(x, y), g^{(n-1)}(y)\right)
$$

Equivalently, $\tilde{F}$ is a quasiproduct map of $X \times Y$. 
We now specialize the above general situation to the particular case of period doubling maps. We first give some definitions and fix some more notations. There are by now several proofs of existence of a regular unimodal function $\varphi$, solution of the Cvitanovic equation

$$
\varphi(\varphi(\varphi(1) \cdot))=\varphi(1) \varphi(\cdot), \quad \varphi(0)=1
$$

See ref. 13 for a review of recent results. We shall denote by $\lambda$ the positive quantity $-\varphi(1)$. This function is analytic in some neighborhood of the interval $[-1,1]$.

More generally, for $\varepsilon>0$ fixed, we shall consider mappings of the interval $[-1,1]$ with the following properties:

P1 $f$ maps the interval $[-1,1]$ into itself.

P2 0 is the only critical point of $f$, and $f(0)=1$.

P3 $f$ has negative Schwartzian derivative, i.e., on the interval $[-1,1]$, $f$ satisfies

$$
\frac{f^{\prime \prime \prime}}{f^{\prime}}-\frac{3}{2}\left(\frac{f^{\prime \prime}}{f^{\prime}}\right)^{2} \leqslant 0
$$

P4 There is a positive number $\varepsilon$, a complex neighborhood $\mathscr{D}$ of $[0,1]$, and a function $\bar{f}$ analytic in $\mathscr{D}$ such that

$$
f(x)=\widetilde{f}\left(|x|^{1+\varepsilon}\right) \quad \text { for } \quad x \in[-1,1]
$$

We shall be mostly interested in the two cases $\varepsilon$ small (see Appendix B) and $\varepsilon=1$, the last one corresponding to analytic even functions.

For a fixed $\varepsilon$ and a fixed domain $\mathscr{D}$, we shall denote by $\mathscr{B}_{1}$ the closure, in the $L^{2}$ space of the Lebesgue measure of $\mathscr{D}$, of the set of bounded analytic functions in $\mathscr{D}$ satisfying $f(0)=1$. Note that this is a space of analytic functions which contains the fixed point associated function $\bar{\phi}$. Moreover, $\mathscr{D}$ is mapped strictly into itself by $z \rightarrow \widetilde{\phi}\left(\lambda^{1+\varepsilon} z\right)^{1+\varepsilon}$, where the power is defined continuously starting from the positive real axis. We also observe that we can find in $\mathscr{B}_{1}$ a neighborhood of $\tilde{\phi}$ such that all elements satisfy the hypotheses P1-P4. We shall denote by $\mathscr{B}$ such a neighborhood. The standard renormalization transformation is defined in $\mathscr{B}$, but is not in general an endomorphism.

We introduce now the noise in the iteration as explained above. From now on we consider maps $F(\cdot, \cdot)$ which belong to $\mathscr{B}$ in their first variable. This space will be denoted by $\mathscr{A}$. We shall need to impose later some more regularity on the second variable also. 
We now define the renormalization transformation. As usual, this transformation acts on $\mathscr{A}$, and is composed of iteration and scaling. The purpose of the scaling is to maintain the property P2. Here, however, the situation is slightly more complex, and the inner scaling has to be chosen differently from the outer scaling to ensure adequate cancellations of the scaling factors when the mapping is iterated, i.e.,

$$
\mathfrak{R}_{g}(F)(x, y)=(F(1, g(y)))^{-1} F\left(F\left(F\left(1, g^{-1}(y)\right) x, y\right), g(y)\right)
$$

Our choice of these scaling factors also works for the iteration of the renormalization transformation. This is easy to verify on the following formula:

$$
\lambda_{1}(y) F^{\left[2^{n}\right]}\left(\lambda_{2}(y) x, y\right)=\mathfrak{R}_{g^{2^{n-1}}} 。 \mathfrak{R}_{g^{2^{n}-2}} \circ \ldots \circ \mathfrak{R}_{g^{2}} \circ \mathfrak{R}_{g}(F)(x, y)
$$

where we have denoted by $g^{p}$ the $p^{\text {th }}$ iterate of $g: g^{p}=g \circ \cdots \circ g$ ( $p$ times) and $\lambda_{1}(y)$ and $\lambda_{2}(y)$ are two scaling factors that can be easily written in terms of the successive renormalizations of $F$.

Some care has to be taken for the domain of the renormalization transformation. We shall later assume that some topology has been given on $Y$, and we shall denote by $\mathscr{A}_{0}$ the set of continuous functions from $Y$ to $\mathscr{B}$ equipped with the uniform distance. We note that every element of a small enough neighborhood (in $\mathscr{A}_{0}$ ) of the constant function $\tilde{\phi}$ satisfies the above four hypotheses. In particular, such a neighborhood is contained in the domain of the renormalization transformation.

Formula (5) shows how the renormalization is acting at the noise level. A very important fact is that it is not the noise which is renormalized, but the time evolution $g$ of the noise. This is a very natural situation if we think of the noise as another dynamical variable. The renormalization should indeed act on the time evolution and not on the noise itself, which is a point in phase space. The study of the renormalization is now the study of the iterates of a new skew product dynamical system. A particular realization of the noise is given by an element of $Y$, the renormalization at the noise level is simply the composition of $g$ with itself, and the mapping which is acting on $\mathscr{A}_{0}$ is given by $\Re_{g}$. This new system has an interesting property. Let $F_{0}$ be the random mapping of our interval defined by

$$
F_{0}(x, y)=\varphi(x)
$$

Notice that this map is independent of the noise. It is easy to verify that $F_{0}$ is a fixed point of all the renormalization transformations $\Re_{g}$. It follows easily from the properties of $\varphi$ that the renormalization transformation is well defined in a small enough neighborhood (in $\mathscr{A}_{0}$ ) of $F_{0}$. The 
renormalization maps are also differentiable in a small neighborhood of $F_{0}$, and we have the following formula for the differential of $\Re_{g}$ at $F_{0}$ :

$$
D \Re_{g}\left(F_{0}\right)(H)(x, y)=A H(x, g(y))+B H(x, y)+C H\left(x, g^{-1}(y)\right)
$$

where $A, B$, and $C$ are three (linear) operators acting on the tangent space $\mathscr{E}$ to $\mathscr{B}$ at $\varphi$ and defined by

$$
\begin{aligned}
& A K(x)=[K(1) \varphi(x)-K(\varphi(\lambda x))] / \lambda \\
& B K(x)=-\varphi^{\prime}(\varphi(\lambda x)) K(-\lambda x) / \lambda \\
& C K(x)=-x \varphi^{\prime}(x) K(1) / \lambda
\end{aligned}
$$

where $K$ is a function in $\mathscr{E}$. It is a very remarkable fact that the linearized renormalization has such a special form and in particular that the action on the noise is almost disconnected from the action on the phase space variable. As we shall see, this will greatly simplify the analysis.

The linear renormalization analysis is now the study of the asymptotic behavior of the following product of linearized renormalization transformations (compare with Eq. (5)):

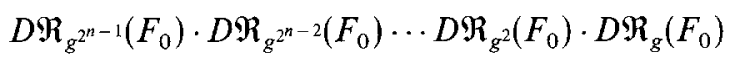

The previous product can be written in a more compact form if we introduce the unitary operator $U$ associated with the time evolution $g$ of the noise (recall that $g$ is invertible and measure-preserving). This operator is acting on $L^{2}(Y, d v)$ and is given by

$$
U \psi(y)=\psi(g(y))
$$

If we introduce the operator $\mathscr{L}_{U}$ given by

$$
\mathscr{L}_{U}=U A+B+U^{-1} C
$$

the product in Eq. (9) can also be written

$$
\mathscr{L}_{U^{2^{n-1}}} \cdot \mathscr{L}_{U^{2^{n-2}}} \cdot \mathscr{L}_{U^{n}} \cdot \mathscr{L}_{U}
$$

\section{ANALYSIS OF THE LINEAR RENORMALIZATION}

As we shall see, the asymptotic behavior of the linear renormalization can be analyzed in terms of a simpler problem. To describe this problem, we first introduce the operator $L_{\omega}$ acting on $\mathscr{E}$ and given by

$$
L_{\omega}=e^{i \omega} A+B+e^{-i \omega} C
$$


It is easy to verify that for any fixed $\omega, L_{\omega}$ is a compact operator in $\mathscr{E} .{ }^{(7)}$ Let $\mathscr{R}$ denote the transformation of the circle given by

$$
\mathscr{R}(\omega)=2 \omega \bmod (2 \pi)
$$

It is well known that this is a uniformly expanding transformation of the circle. Given an ergodic invariant measure, we can apply the OseledecRuelle theorem ${ }^{(22)}$ to the family of maps $L_{\omega j}$. We obtain an almost everywhere constant maximal Lyapunov exponent (exponential rate of growth) for the product of operators

$$
L_{\mathscr{R}^{n}(\omega)} \cdot L_{\mathscr{Z}^{n-1}(\omega)} \cdots L_{\mathscr{Z}(\omega)} \cdot L_{\omega}
$$

This Lyapunov exponent depends on the ergodic invariant measure which is considered. The map $\mathscr{R}$ of the circle has many invariant measures. We shall be interested in two particular ones (although it may be interesting to consider other cases such as periodic points). The first one is the delta measure at $\omega=0$. For this measure, we have $\omega$ almost surely $L_{\omega}=$ $A+B+C$, which does not depend on $\omega$. From the explicit formulas for $A$, $B$, and $C$, it is easy to verify that the operator $A+B+C$ is the usual linear renormalization operator for maps of the interval (in the absence of noise). Therefore the maximal Lyapunov exponent in this case is equal to $\log \delta$, where (for $\varepsilon=1$ ) $\delta$ is the universal number $4.6692 \ldots$. . If we use the Lebesgue measure instead of the Dirac measure, we expect to find a different Lyapunov number.

We shall denote this number by $\gamma$. We recall that as a consequence of the Oseledec-Ruelle theorem, there is a subset $\mathscr{I}$ of the circle which is invariant under $\mathscr{R}$, of Lebesgue measure $2 \pi$, and such that for any $\omega$ in $\mathscr{I}$ the product of operators (14) grows with an exponential rate $\gamma$ outside of a linear subspace of codimension at least one. Using a method developed by Herman, ${ }^{(18)}$ one can give a lower bound on $\gamma$. First, one can show, as in ref. 6, that, except for an exponent $-2 \log \hat{\lambda}$, the operators $L_{\omega}$ and

$$
M_{\omega} K(x)=-\left[K(\phi(\lambda x)) e^{i \omega}+\phi^{\prime}(\phi(\lambda x)) K(-\lambda x)\right] / \lambda
$$

have the same Lyapunov exponents. This last family of operators is now acting on functions which are not restricted to have the value zero for $x=0$. It is now easy to apply Herman's lower bound estimate to get that $\gamma \geqslant-2 \log \lambda$. Numerical simulations (for $\varepsilon=1$ ) give a value around 6.4.

We now come back to the analysis of the asymptotic behavior of the product (11). Let $\mathscr{H}$ denote the Hilbert space $L^{2}(Y, d v)$. This Hilbert space can be disintegrated with respect to the Abelian von Neumann algebra generated by the unitary operator $U \cdot{ }^{(11)}$ As we shall see below, this is a convenient way to do the spectral analysis of $U$. There is a measure $\rho$ on 
the circle $S^{1}$ and a measurable field of Hilbert spaces $\mathscr{H}_{\omega}$ (depending on $U$ ) such that

$$
\mathscr{H}=\int_{S^{1}}^{\oplus} \mathscr{H}_{\omega} d \rho(\omega)
$$

The operator $U$ is disintegrated into the field of operators

$$
U_{\omega}=e^{i \omega} \mathbf{I}_{\omega}
$$

where $\mathbf{I}_{\omega}$ is the identity operator in $\mathscr{H}_{\omega}$. Similarly, we can disintegrate the Hilbert space $\mathscr{F}$ defined by

$$
\mathscr{F}=\mathscr{E} \otimes \mathscr{H}
$$

into a field of Hilbert spaces $\mathscr{F}_{\omega}$. The operator $\mathscr{L}_{U}$ which acts on $\mathscr{F}$ according to $(10)$ is disintegrated into a field of operators given by

$$
\left(\mathscr{L}_{U}\right)_{\omega}=e^{i \omega} A+B+e^{-i \omega} C
$$

We can construct similarly a global operator $\mathscr{M}_{U}$ on $\mathscr{F}$, which is disintegrated into the field of operators $\left(M_{\omega}\right)_{\omega \in S^{1}}$ defined in (15).

Moreover, $U^{2^{n}}$ obviously disintegrates to $e^{i 2^{n} \omega_{\omega}} \mathbf{I}_{\omega}$. At this point, we cannot apply the Oseledec-Ruelle theorem to conclude about the growth rate of the product of operators in Eq. (11). The reason is that the above theorem only gives the asymptotic behavior of the product (14) for a fixed $\omega$. The transient behaviors may vary wildly with $\omega$ and this nonuniformity can change the asymptotic behavior of the integrated product (11). Such a phenomenon can actually be shown explicitly for the easier problem of the influence of noise on the intermittency transition. It is also reflected in the difference between the above number and the universal number found for the case of independent noise.

We emphasize that the components $\left(\mathscr{L}_{U}\right)_{\omega}$ do not depend on the time evolution $g$. Therefore, the asymptotic behavior of $\mathscr{L}_{U}$ will depend on $g$ only through the associated spectral decomposition. We shall identify $L_{\omega}$ and $\left(\mathscr{L}_{U}\right)_{\omega}$, since these operators are identical for all noises, except for their multiplicity.

Essential differences between various types of noises appear in the asymptotic behavior of $\mathscr{L}_{U}$. The case of a quasiperiodic noise is treated below (Theorem 3.3). We shall show at the end of this section how to obtain the critical index for a noise with Lebesgue spectrum.

We now consider the particular case of quasiperiodic noise. In this case, $Y=S^{1}$ (endowed with the Lebesgue measure), and the disintegration can be done by Fourier transformation. Time evolution of the noise is 
simply a rotation. More precisely, for a given rotation angle $\omega$, the linear renormalization is acting on functions $H$ of $x$ and the angle $y$ by the formula

$$
\left(D \Re_{\omega} H\right)(x, y)=A H(x, y+\omega)+B H(x, y)+C H(x, y-\omega)
$$

(see Eq. (7)). In order to simplify notations, we shall denote by $\mathscr{L}_{\omega}$ the operator $D \Re_{\omega}$. The above disintegration is simply the Fourier transform with respect to $y$, and we are led to the family of operators

$$
\left(\mathscr{L}_{\omega} H\right)_{n}(x)=e^{i n \omega} A H_{n}(x)+B H_{n}(x)+e^{-i n \omega} C H_{n}(x)
$$

where $n$ is the integer variable conjugated to $y$. By Fourier transformation the operator $\mathscr{L}_{\omega}$ has been decomposed into a (countable) direct sum of operators. Each of these operators is now of the form $L_{n \omega}$, and we first have to compute the Lyapunov exponent for each Fourier sector separately. Consider first the sector $n=0$. In this case, as we have previously observed, the tangent operator is the linearized renormalization without noise, and the Lyapunov exponent is the logarithm of the universal number $\delta$. If we consider the case $n=1$ and choose $\omega$ in $\mathscr{I}$ (which is a set of full measure in $S^{1}$ ), we get a Lyapunov number equal to $\gamma$. Consider now the case $n=2$. In order to obtain an exponent equal to $\gamma$, we have to take $2 \omega \in \mathscr{I}$. But it is easy to verify that the set $\{\omega \mid 2 \omega \in \mathscr{I}\}$ is also of full measure. More generally, the set $\tilde{\mathscr{I}}$ given by

$$
\tilde{I}=\{\omega \mid p \omega \in \mathscr{I} \forall p \in \mathbf{Z} \backslash\{0\}\}
$$

is a set of full measure. Therefore, if $\omega$ belongs to $\tilde{\mathscr{I}}$, we observe the Lyapunov number $\gamma$ in all Fourier sectors except the zero sector.

At this point, we can observe a situation which is new with respect to the standard renormalization group analysis. Each Fourier sector (except $n=0$ ) possesses one unstable direction and our fixed point (6) is now of codimension infinity. In other words, one needs to impose a countable number of constraints to observe a complete sequence of period doubling bifurcations in the presence of quasiperiodic noise. If this is not the case, one will be able to distinguish only finitely many such bifurcations on the bifurcation diagram. Through the renormalization, the system flows away from the fixed point (6) and it would be interesting to understand the nature of this crossover, which may be nontrivial even for simple noises like rational rotations (see ref. 17 for results in this direction).

We now come to the question of the asymptotic behavior of the integrated product (11). Although, as we explained before, every Fourier sector (except the zero sector) has the same Lyapunov exponent, this number may not be the global Lyapunov exponent. Various counter- 
examples can be constructed easily using lacunar series. More precisely, an element $H$ of the tangent space can be decomposed in a sequence of Fourier components $\left(H_{n}\right)_{n \in \mathbf{Z}}$, and we have

$$
\left\|\mathscr{L}_{\omega}^{[q]} H\right\|^{2}=\sum_{n}\left\|L_{n \omega}^{[q]} H_{n}\right\|^{2}
$$

Each term in the sum (except $n=0$ ) behaves exponentially like $e^{2 q \gamma}$; however, this asymptotic behavior is not reached at a simultaneous speed for each term and this can upset the large- $q$ behavior of the logarithm of the sum. In order to control this phenomenon, we need a precise estimate of the quantities

$$
e^{-q \gamma}\left\|L_{n \omega}^{[q]} H_{n}\right\|
$$

for various $n$ 's and $\omega$ 's. This estimate is provided by the large-deviation theory of the largest Lyapunov exponent developed in Appendix A. We shall assume that the hypotheses HA.1 and HA.2 of this appendix are satisfied. In Appendix B, we show that this is indeed the case in the situation where the $\varepsilon$ expansion can be controlled. For the natural case $\varepsilon=1$, it should be possible to make a computer-assisted proof of these two hypotheses. We shall also assume from now on that $\gamma$ is larger than $\log \delta$ (this is true at least in $\varepsilon$ expansion, and follows numerically, in the case $\varepsilon=1$, from the lower bound $\gamma \geqslant-2 \log \lambda$ ). We shall denote by $\mathscr{C}_{p}$ for $p \in \mathbf{N}$ the Hilbert space of maps from $S^{1}$ to $\mathscr{E}$ such that the $\mathscr{E}$ norm of their first $p$ derivatives is square integrable with respect to the normalized Lebesgue measure $l$ of $S^{1}$. It is easy to verify that for any angle $\omega, \mathscr{L}_{\omega}$ is a continuous operator in $\mathscr{C}_{p}$. We shall simply denote $\mathscr{C}_{0}$ by $\mathscr{C}$. We first have the following trivial lower bound.

Lemma 3.1. If $\omega \in \tilde{\mathscr{I}}$, there is an infinite-codimension vector space $\mathscr{C}_{\omega}$ in $\mathscr{C}$ such that if $H \in \mathscr{C}-\mathscr{C}_{\omega}$, then

$$
\liminf _{q \rightarrow \infty} q^{-1} \log \left\|\mathscr{L}_{\omega}^{[q]} H\right\| \geqslant \gamma
$$

Proof. The result follows at once from the above considerations, since it is enough to have the exponent $\gamma$ in one Fourier sector to ensure the lower bound.

We shall now prove an upper bound.

Proposition 3.2. There is a subset $\Theta$ of $\tilde{\mathscr{I}}$ of full measure and a positive constant $c_{3}$ such that if $\omega \in \Theta$ and if for some positive number $\eta$ large enough, $H$ satisfies for every $n \in \mathbf{Z}$

$$
\left\|H_{n}\right\| \leqslant \mathcal{O}(1)\left(1+n^{2}\right)^{-\eta / 2}
$$


then

$$
\limsup _{q \rightarrow \infty} q^{-1} \log \left\|\mathscr{L}_{\omega}^{[q]} H\right\| \leqslant \gamma+c_{3} \eta^{-1 / 2}
$$

Proof. We first lift the map $\omega \rightarrow L_{\omega}$ from $S^{1}$ to $\{0,1\}^{\mathrm{z}}$ using the dyadic representation of $\omega \in S^{1}$. Since the new map $\Omega \rightarrow L_{\Omega}, \Omega \in\{0,1\}^{Z}$ does not depend on the elements of $\Omega$ with negative indices, the hypotheses of Appendix A are still satisfied. Given an angle $\omega$, there is of course a countable number of elements of $\{0,1\}^{\mathbf{Z}}$ which project onto it. We shall denote by $p$ the map which associates to any $\Omega$ in $\{0,1\}^{z}$ the angle $\omega$ in $S^{1}$ whose dyadic expansion is given by the coefficients with positive index of $\Omega$. We shall not mention explicitly the preimage in statements which refer to all the preimages of a given angle.

We now define several sequences of sets which shall be useful in the definition of $\Theta$. For any positive integer $q$, we first define a real function $S^{q}$ on $\{0,1\}^{\mathbf{z}}$ by

$$
S^{q}(\Omega)=\sum_{0}^{q-1}\left(\log \rho_{\mathscr{S}_{S} \Omega}-\gamma\right)
$$

where $\rho_{\Omega}$ is defined in Appendix A. We first observe that $S^{q} \leqslant q A$, where

$$
A=\sup _{\Omega}\left(\log \rho_{\Omega}-\gamma\right)>0
$$

If $\varepsilon$ is a positive number, we define a set $\Xi_{q}^{\varepsilon}$ by

$$
\Xi_{q}^{\varepsilon}=\left\{\omega \mid \forall \Omega \in p^{-1}(\omega), S^{q}(\Omega) \leqslant q \varepsilon\right\}
$$

From the large-deviation Lemma A.6 of Appendix A, we deduce that there are positive constants $B, \varepsilon_{0}$, and an integer-valued function $q(\cdot)$ such that if $0 \leqslant \varepsilon \leqslant \varepsilon_{0}$ and $q>q(\varepsilon)$, we have

$$
l\left(\Xi_{q}^{\varepsilon}\right) \geqslant 1-e^{-B q \varepsilon^{2}}
$$

We now choose

$$
\varepsilon=\left(\frac{2 A}{B(\eta-2)}\right)^{1 / 2}
$$

and $\eta$ large enough so that $\varepsilon<\varepsilon_{0}$. We define a set of angle $\Theta_{q}^{\eta}$ by

$$
\Theta_{q}^{\eta}=\bigcap_{|n|<\exp [A q /(\eta-2)]}\left\{\omega \mid n \omega \in \Xi_{q}^{\varepsilon}\right\}
$$


From the previous estimates and definitions, it is easy to verify that for $\eta$ large enough and $q$ larger than $q(\varepsilon)$, we have

$$
l\left(\Theta_{q}^{\eta}\right) \geqslant 1-2 e^{A q /(\eta-2)} e^{-2 q A /(\eta-2)} \geqslant 1-2 e^{-q A /(\eta-2)}
$$

We now define a set $\Theta^{n}$ by

$$
\Theta^{\eta}=\bigcup_{p=q(\varepsilon)}^{\infty} \bigcap_{l=p}^{\infty} \Theta_{l}^{\eta}
$$

It follows immediately that

$$
l\left(\Theta^{\eta}\right)=1
$$

If $\omega$ belongs to $\Theta^{\eta}$, we have $\omega \in \Theta_{q}^{\eta}$ for any $q$ large enough. Therefore, from Lemma A.5,

$$
\begin{aligned}
\left\|\mathscr{L}_{\omega}^{[q]} H\right\|^{2} \geqslant & \mathcal{O}(1) \sum_{|n|<\exp [q A /(\eta-2)]}\left\|H_{n}\right\|^{2} \prod_{0}^{q-1} \rho_{p^{-1}\left(n p\left(\mathscr{S}^{\prime}\right)(\Omega)\right)}^{2} \\
& +\mathscr{O}(1) \sum_{\exp [q A /(\eta-2)] \leqslant|n|}\left\|H_{n}\right\|^{2} e^{2 q(A+\gamma)} \\
\leqslant & \mathcal{O}(1) e^{2 q(\gamma+\varepsilon)}\|H\|^{2}
\end{aligned}
$$

for any $q$ large enough. This proves that on $\Theta^{\eta}$, the maximal Lyapunov exponent, is smaller than $\gamma+\mathcal{O}(1) \eta^{-1 / 2}$. Note that instead of using the $L^{2}$ norm, we can derive a similar result using any Sobolev norm.

Theorem 3.3. There is a subset $\Theta$ of $\tilde{\mathscr{I}}$ of full measure such that if $\omega$ is an element of $\Theta$, if $H$ is a nonzero $C^{\infty}$ function in $\mathscr{C}$ which does not belong to the (infinite-codimension) subspace $\mathscr{C}_{\omega}$, then

$$
\lim _{q \rightarrow \infty} q^{-1} \log \left\|\mathscr{L}_{\omega}^{[q]} H\right\|=\gamma
$$

In other words, we have to impose some regularity on the $y$ dependence of $H$ to be able to use the large-deviation results and to prevent the occurrence of another Lyapunov exponent. For $C^{\infty}$ functions we get exactly the Lyapunov exponent.

Proof. We define a set $\Theta$ of full measure by

$$
\Theta=\bigcap_{r>2+2 A / B \varepsilon_{0}^{2}}^{\infty} \Theta^{r}
$$


If $H$ is a $C^{\infty}$ function and if $\omega \in \Theta$, we can apply Proposition 3.2 for all values of $\eta$ which are large enough integers. The result follows now from Lemma 3.1.

As remarked before, the same result holds if we replace the $L^{2}$ norm by a Sobolev norm (in some $\mathscr{C}_{p}$ ).

We now come back to the case of noises with Lebesgue spectrum. Since the spectral measure introduced in (16) is in fact a class of equivalent measures on $S^{1}$, we can assume that the spectral measure of such noises in the (normalized) Lebesgue measure on $S^{1}$.

We recall that the linearized renormalization group result for Gaussian noises can be formulated as follows.

Theorem 3.4. There is a linear subspace $\mathscr{F}_{G}$ of $\mathscr{F}$ of codimension at least one such that if $H \in \mathscr{F}-\mathscr{F}_{G}$, we have

$$
\lim _{n \rightarrow \infty} n^{-1} \log \left\|\mathscr{M}_{U}^{[n]} H\right\|=\gamma_{G}
$$

where $\gamma_{G}$ is a universal number. Moreover, in $\mathscr{F}_{G}$ the limit is smaller.

This result was first proven for independent Gaussian noises, ${ }^{(8,9,19,21,23)}$ and can be extended to correlated Gaussian noises. ${ }^{(15)}$ The universal number $\gamma_{G}$ depends on $\varepsilon$ and for $\varepsilon=1$ its value is about $6.6192 \ldots$. For small $\varepsilon$, one gets $\gamma_{G}=\log (\sqrt{2} / \lambda)+\mathcal{O}(1)$.

One can give a proof of Theorem 3.4 using the general technique of renormalization explained above. It is, however, a tedious rephrasing of most of the above arguments with some adaptations, and we only present here the interesting part, which is an expression for the number $\gamma_{G}$.

Theorem 3.5. The universal number $\gamma_{G}$ is given by

$$
\gamma_{G}=\Phi(2) / 2
$$

where $\Phi$ is the free energy of the Lyapunov exponent defined in Appendix A.

The first step in the proof of Theorem 3.5 is an elementary relation between $L_{\omega}$ and $M_{\omega}$.

Lemma 3.6. For any $n \geqslant 1$ and any $\omega \in S^{1}, M_{\omega}^{[n]}-L_{\omega}^{[n]}$ is an operator of rank one on $\mathscr{E}$, and we have

$$
M_{\omega}^{[n]} h=L_{\omega}^{[n]} h-\lambda^{-1} a_{2^{n} \omega} \sum_{0}^{n-1} e^{-i 2^{p+1} \omega} L_{\omega}^{[p]} h(1) \quad \forall h \in \mathscr{E}
$$

where $a_{\omega}(x)=e^{2 t \omega} \phi(x)-x \phi^{\prime}(x)$ satisfies $M_{2 \omega} a_{\omega}=a_{2 \omega}$. 
We now consider a subset $\mathscr{F}_{P}$ of $\mathscr{F}$ defined by

$$
\mathscr{F}_{P}=\left\{H \in \mathscr{F} \mid H(x, y)=h(x) k(y) \text { with } h \in \mathscr{E}, \text { and } k \in L^{2}(Y, d v)\right\}
$$

It is easy to show that $\mathscr{F}_{P}$ is total in $\mathscr{F}$, and therefore one can find an element $H=h k$ of $\mathscr{F}_{P}$ which does not belong to $\mathscr{F}_{G}$.

It is known that one can make even more restrictive assumptions on $H$. We recall that the space $L^{2}(Y, d v)$ is an infinite tensor product of spaces $L^{2}\left(\mathbf{R}, e^{-x^{2}} d x / \sqrt{\pi}\right)$, and it is enough to consider a function $k$ which depends only on one component. ${ }^{(15)}$ One can still find couples $h$ and $k$ such that Eq. (20) still holds for $H=h k$. Moreover, this is true in the complement of a linear space of codimension at least one of the linear space of functions $h$ in $\mathscr{E}$. It is easy to obtain for such vectors $h k$ the expression of the spectral disintegration, and we have

$$
\left\|\mathscr{M}_{U}^{[n]}(h k)\right\|^{2}=\|k\|^{2} \int_{S^{1}}\left\|M_{\omega}^{[n]} h\right\|^{2} d \omega
$$

The inequality

$$
\gamma_{G} \leqslant \Phi(2) / 2
$$

follows at once from Theorem 3.4, Lemma 3.6, and the results of Appendix A (in particular Lemma A.5).

We now prove the lower bound. It follows easily from Lemma A.5 that we can replace $L_{\omega}^{[n]} h$ by

$$
\left\langle\alpha_{\Omega} \mid h\right\rangle e_{\mathcal{S}^{n} \Omega} \prod_{0}^{n-1} \rho_{\mathscr{P}_{\Omega} \Omega}
$$

where we have used again the extension to $\{0,1\}^{\mathrm{z}}$ of the dyadic representation. It follows now from the continuity of $\alpha_{\Omega}$ and the expansivity of the map

$$
\omega \rightarrow 2 \omega \bmod (2 \pi)
$$

that if there is an $\Omega$ such that $\left\langle\alpha_{\Omega} \mid h\right\rangle \neq 0$, then

$$
\Phi(2) / 2=\lim (2 n)^{-1} \log \int_{S^{1}}\left\|L_{\omega}^{[n]} h\right\|^{2} d \omega
$$

Therefore, using Lemma 3.6, we conclude that if $\gamma_{G}$ is strictly smaller than $\Phi(2) / 2$, some cancellation should occur between $a_{2^{n} \omega}$ and a multiple of $e_{\mathscr{S}^{n} \Omega}$ at least for large $n$. This is, however, impossible because both are analytic 
functions in $\mathscr{D}$ of norm $\mathscr{O}(1)$, and moreover, $e_{\mathscr{y}^{n} \Omega}(0)=0$, while $a_{2^{n} \omega}(0)=$ $\exp \left(i 2^{n+1} \omega\right)$.

We conclude with a comparison of the critical indices for different noises, the index $\varepsilon$ of the universality class with respect to $x$ [cf. (3)] being fixed (the thermodynamic limit $\Phi$, hence the indices $\gamma$ and $\gamma_{G}$, will of course depend on $\varepsilon$ ). Theorem 3.4 shows that the critical exponent for a noise with Lebesgue spectrum is equal to $\Phi(2) / 2$, which is the value for an independent Gaussian noise. The critical exponent for a quasiperiodic noise, which is equal to $\Phi^{\prime}(0)$, is different, at least in the $\varepsilon$ expansion. However, the critical index does not depend on the details of the noise considered; it is universal within each class of noises of the same spectral type (and for adequate functions, as explained in Theorems 3.3 and 3.4).

\section{APPENDIX A. LARGE DEVIATIONS OF THE MAXIMAL LYAPUNOV EXPONENT}

Here we shall present the theory of large deviations for the maximal Lyapunov exponent in a situation which is more general than what is needed for our applications. Similar results were proven in one dimension by Bohr and Rand. ${ }^{(4)}$ The argument is also very similar to the proof of the volume lemma. ${ }^{(3)}$ We shall denote by $E$ the set $\{0,1\}^{\mathrm{Z}}$ equipped with the usual dyadic distance. $l$ will denote the probability measure which is the infinite product of the measure with weight $1 / 2$ on each atom of $\{0,1\}$. We shall denote by $p$ the map which associates to any $\Omega$ in $\{0,1\}^{\mathrm{z}}$ the angle $\omega$ in $S^{1}$ whose dyadic expansion is given by the coefficients with positive index of $\Omega$.

Note that all the results below can be easily extended to the case of shift-invariant, short-range Gibbs state on a subshift of finite type. Let $\mathscr{E}$ be a complex separable Hilbert space. The associated scalar product will be denoted by $\langle\mid\rangle$. We shall assume that a (uniformly) Hölder continuous map

$$
\Omega \rightarrow t_{\Omega}
$$

is given from $E$ to the unit sphere of $\mathscr{E}$, and also a (uniformly) Hölder continuous map

$$
\Omega \rightarrow L_{\Omega}
$$

from $E$ to the set of compact operators on $\mathscr{E}$. For a given positive number $a$ larger than 1 , we shall denote by $C_{a, \Omega}$ the cone

$$
C_{a, \Omega}=\left\{h \in \mathscr{E}|\|h\| \leqslant a|\left\langle h \mid t_{\Omega}\right\rangle \mid\right\}
$$

We make the following hypotheses. 
HA1. There is a positive number $a^{\prime}$ with $1<a^{\prime}<a$ such that for all $\Omega$ in $E$, we have

$$
L_{\Omega} C_{a, \Omega} \subset C_{a^{\prime}, \mathscr{S} \Omega}
$$

HA2. There is a positive number $b$ such that

$$
\inf _{\substack{h \in C_{a, \Omega} \\\left|\left\langle h \mid t_{\Omega}\right\rangle\right|=1}}\left|\left\langle t_{\mathscr{S} \Omega} \mid L_{\Omega} h\right\rangle\right| \geqslant b>0
$$

Similar hypotheses can be used to deal with the case of a real cone. One then has to use Hilbert's projective metric instead of the complex projective metric defined below (see ref. 16 for a related result). We define two positive real numbers by $A=\left(a^{2}-1\right)^{1 / 2}$ and $A^{\prime}=\left(a^{\prime 2}-1\right)^{1 / 2}$. We shall denote by $d_{C, \Omega}$ the Caratheodory-Reiffen distance on the ball of radius $A$ in $t_{\Omega}^{\perp}$ (see ref. 14 for a definition). We recall that if $A^{\prime \prime}<A$, there is a positive number $K$ (depending on $A$ and $A^{\prime \prime}$ ) such that if $u_{1}$ and $u_{2}$ belong to $t_{\Omega}^{\perp}$ and have norms less than $A^{\prime \prime}$, then

$$
\left\|u_{1}-u_{2}\right\| \leqslant d_{C, \Omega}\left(u_{1}, u_{2}\right) \leqslant K\left\|u_{1}-u_{2}\right\|
$$

We now define a projective distance $d_{\Omega}$ on $C_{a, \Omega}$ by

$$
d_{\Omega}\left(h_{1}, h_{2}\right)=d_{C, \Omega}\left(\frac{h_{1}}{\left\langle t_{\Omega} \mid h_{1}\right\rangle}-t_{\Omega}, \frac{h_{2}}{\left\langle t_{\Omega} \mid h_{2}\right\rangle}-t_{\Omega}\right)
$$

The following result is the main reason for introducing this projective metric.

Proposition A.1. If the hypothesis HA1 above is satisfied, for any $\Omega$ there is a positive number $\theta_{\Omega}$ such that

$$
d_{\mathscr{S} \Omega}\left(L_{\Omega} h_{1}, L_{\Omega} h_{2}\right) \leqslant \theta_{\Omega} d_{\Omega}\left(h_{1}, h_{2}\right)
$$

for any pair $h_{1}, h_{2}$ of vectors in $C_{a, \Omega}$. Moreover, $\theta=\sup _{\Omega} \theta_{\Omega}<1$.

Proof. We can represent $L_{\Omega}$ from the decomposition $\mathscr{E}=\mathbf{C} t_{\Omega} \oplus t_{\Omega}^{\perp}$ into $\mathscr{E}=\mathbf{C} t_{\mathscr{S} \Omega} \oplus t_{\mathscr{S} \Omega}^{\perp}$ by a matrix:

$$
L_{\Omega}=\left(\begin{array}{ll}
L_{1,1}(\Omega) & L_{1,2}(\Omega) \\
L_{2,1}(\Omega) & L_{2,2}(\Omega)
\end{array}\right)
$$

We now define a function $J_{\Omega}$ of the ball of radius $A$ in $t_{\Omega}^{\perp}$ by

$$
J_{\Omega}(h)=\frac{L_{2,1}(\Omega)+L_{2,2}(\Omega) h}{L_{1,1}(\Omega)+L_{1,2}(\Omega) h}
$$


From our hypotheses, $J_{\Omega}$ is an analytic map from the ball of radius $A$ (in $t_{\Omega}^{\perp}$ ) in the ball of (smaller) radius $A^{\prime}$ (in $t_{\mathscr{S}_{\Omega}}^{\perp}$ ), hence the iterates of $J_{\Omega}$ are well defined. The result now follows from ref. 14 .

Our next result provides information about the field of unstable directions.

Proposition A.2. There is a (uniformly) Hölder continuous map $\Omega \rightarrow e_{\Omega}$ from $E$ to $\mathscr{E}$ such that $e_{\Omega}$ belongs to $C_{a^{\prime}, \Omega},\left\langle e_{\Omega} \mid t_{\Omega}\right\rangle=1$, and

$$
L_{\Omega} e_{\Omega}=\tilde{\rho}_{\Omega} e_{\mathscr{S} \Omega}
$$

where the function $\Omega \rightarrow \tilde{\rho}_{\Omega}$ from $E$ to $\mathbf{C}$ is (uniformly) Hölder continuous and has modulus $\rho_{\Omega}$ larger than $b$.

Proof. It follows from Proposition A.1. that the sequence

$$
J_{\mathscr{S}-q_{\Omega}}^{[q]}(0)
$$

converges exponentially fast in the Caratheodory-Reiffen metric to a vector $u_{\Omega} \in t_{\Omega}^{\perp}$. We denote by $e_{\Omega}$ the vector $t_{\Omega}+u_{\Omega}$. It is easy to verify that $e_{\Omega}$ belongs to $C_{a^{\prime}, \Omega}$ and also that its image by $L_{\Omega}$ is proportional to $e_{\mathscr{S} \Omega}$. The proportionality factor will be denoted by $\tilde{\rho}_{\Omega}$, namely

$$
\tilde{\rho}_{\Omega}=\left\langle t_{\mathscr{S}_{\Omega}} \mid L_{\Omega} e_{\Omega}\right\rangle
$$

From the hypothesis $\mathbf{H A 2}$ we have $\rho_{\Omega} \geqslant b$. Note that $\rho_{\Omega}$ is also uniformly bounded above since $L_{\Omega}$ is uniformly bounded. It now remains to show that $e_{\Omega}$ is Hölder continuous. The Hölder continuity of $\rho_{\Omega}$ will then follow from the above formula. Let $\Omega$ and $\Omega^{\prime}$ be two elements of $E$. Let $l$ be defined by $d\left(\Omega, \Omega^{\prime}\right)=2^{-l}$. Let $l^{\prime}$ be the largest integer smaller than $l / 2$. We can assume that $l^{\prime}$ is large, otherwise the Hölder estimate follows from the boundedness of $e_{\Omega}$, i.e., we shall assume that $l$ is larger than some integer $l_{0}$ to be chosen later. From the Hölder continuity to $t_{\Omega}$, it follows that for some positive constants $B_{1}$ and $b_{1}$ we have, from $d\left(\mathscr{S}^{-l^{\prime}} \Omega, \mathscr{S}^{-l^{\prime}} \Omega^{\prime}\right) \leqslant 2^{-l^{\prime}}$,

$$
\left|\left\langle t_{\mathscr{S}^{-l} l_{\Omega}} \mid t_{\mathscr{S}-l_{\Omega^{\prime}}}\right\rangle-1\right| \leqslant B_{1} 2^{-l^{\prime} b_{1}}
$$

For $0 \leqslant p \leqslant l^{\prime}\left(l^{\prime}\right.$ fixed), we define three sequences $x_{p}, y_{p}$, and $z_{p}$ by

and

$$
\begin{aligned}
& x_{p}=J_{\mathscr{S}-r^{\prime} \Omega}^{[p]}(0) \\
& y_{p}=J_{\mathscr{S}-l^{\prime} \Omega^{\prime}}^{[p]}\left(\frac{t_{\mathscr{D}-l^{\prime} \Omega}}{\left\langle t_{\mathscr{S}-l^{\prime} \Omega^{\prime}} \mid t_{\mathscr{P}-l^{\prime} \Omega}\right\rangle}-t_{\mathscr{S}-l^{\prime} \Omega^{\prime}}\right)
\end{aligned}
$$

$$
z_{p}=\frac{t_{\mathscr{P} p-i_{\Omega^{\prime}}}+y_{p}}{\left\langle t_{\mathscr{S P} p-I_{\Omega^{\prime}}}+y_{p} \mid t_{\mathscr{P} p-r_{\Omega}}\right\rangle}-t_{\mathscr{P} p-l_{\Omega}}
$$


Note that $z_{p} \in t_{\mathscr{y}^{p-l}}^{\perp}$ and $\left\|z_{p}\right\| \leqslant A^{\prime}$. We also have $z_{p}=y_{p}+\mathcal{O}(1) 2^{-l^{\prime} b_{1}}$. Using the Hölder continuity of $L_{\Omega}$ and the hypothesis HA1, we deduce, for $l_{0}$ large enough, that there are two constants $B_{2}$ and $b_{2}$ (independent of $\Omega$ and $\Omega^{\prime}$ ) such that

$$
\left\|J_{\mathscr{S} p-l^{\prime} \Omega}\left(z_{p}\right)-J_{\mathscr{S}^{p-l} \Omega^{\prime}}\left(y_{p}\right)\right\| \leqslant B_{2} 2^{-l^{\prime} b_{2}}
$$

This implies that for some similar constant $B_{3}$, we have

$$
\left\|z_{p+1}-J_{\mathscr{S}^{p-l^{\prime}} \Omega}\left(z_{p}\right)\right\| \leqslant B_{3} 2^{-l^{\prime} b_{2}}
$$

From the properties of the Caratheodory-Reiffen metric, we have for some new constant $B_{4}$

$$
d_{C, \mathscr{P} p-l^{\prime}+1_{\Omega}}\left(x_{p+1}, z_{p+1}\right) \leqslant \theta d_{C, \mathscr{P} p-l^{\prime} \Omega}\left(x_{p}, z_{p}\right)+B_{4} 2^{-l^{\prime} b_{2}}
$$

and we finally get

$$
d_{C, \Omega}\left(x_{l^{\prime}}, y_{l^{\prime}}\right) \leqslant O(1)\left(\theta^{l^{\prime}}+2^{-l^{\prime} b_{2}}\right)
$$

We also have, using the contraction of the Caratheodory-Reiffen metric,

$$
\left\|e_{\Omega}-t_{\Omega}-x_{l^{\prime}}\right\| \leqslant d_{C, \Omega}\left(e_{\Omega}-t_{\Omega}, x_{l^{\prime}}\right) \leqslant \mathcal{O}(1) \theta^{l^{\prime}}
$$

and a similar estimate for $\Omega^{\prime}$. We finally get

$$
\begin{aligned}
\left\|e_{\Omega}-e_{\Omega^{\prime}}\right\| \leqslant & \left\|e_{\Omega}-t_{\Omega}-x_{l^{\prime}}\right\|+\left\|t_{\Omega}+x_{l^{\prime}}-t_{\Omega}-z_{l^{\prime}}\right\| \\
& +\left\|t_{\Omega}+z_{l^{\prime}}-t_{\Omega^{\prime}}-y_{l^{\prime}}\right\|+\left\|e_{\Omega^{\prime}}-t_{\Omega^{\prime}}-y_{l^{\prime}}\right\| \\
\leqslant & \mathcal{O}(1)\left(\theta^{l^{\prime}}+2^{-l^{\prime} b_{2}}\right)
\end{aligned}
$$

and the result follows from $l^{\prime} \geqslant l / 3$.

The following lemma will be useful later to estimate the growth rate of a general vector.

Lemma A.3. Let $v$ be a vector which does not belong to the cone $C_{a, \Omega}$. Then

$$
\left\|v-e_{\Omega}\left\langle v \mid t_{\Omega}\right\rangle\right\| \geqslant\left(1-\frac{a^{\prime}}{a}\right)\|v\|
$$

Proof. If the reverse (strict) inequality holds for some vector $v$, we have from $\left\|e_{s}\right\| \leqslant a^{\prime}$ the inequality

$$
a\left|\left\langle v \mid t_{\Omega}\right\rangle\right| \leqslant\|v\|\left\langle a^{\prime}\left|\left\langle v \mid t_{\Omega}\right\rangle\right|+c_{1}\|v\|\right.
$$


where $c_{1}=1-a^{\prime} / a$ (the first inequality follows from $v \notin C_{a, \Omega}$ ). This implies

and

$$
\left|\left\langle v \mid t_{\Omega}\right\rangle\right|<\frac{c_{1}\|v\|}{a-a^{\prime}}
$$

$$
\|v\|<c_{1} \frac{a}{a-a^{\prime}}\|v\|=\|v\|
$$

which is a contradiction.

We can now analyze the behavior under iteration of vectors which do or do not belong to the $C_{a, \Omega}$.

Lemma A.4. There is a constant $c_{2}$ such that if $v$ is a vector and $n$ is an integer satisfying $L_{\Omega}^{n} v \notin C_{a, \mathscr{g}^{n} \Omega}$, then

$$
\left\|L_{\Omega}^{n} v\right\| \leqslant c_{2}\|v\| \prod_{0}^{n-1} \theta_{\mathscr{S}_{\Omega} \Omega} \rho_{\mathscr{S}_{\Omega} \Omega}
$$

If $v$ belongs to $C_{a, \Omega}$, then for every integer $n>0$ we have

$$
c_{2}^{-1} \leqslant \frac{\left|\left\langle t_{\mathscr{S}^{n} \Omega} \mid L_{\Omega}^{[n]} v\right\rangle\right|}{\|v\| \prod_{0}^{n-1} \rho_{\mathscr{Y} \Omega_{\Omega}}} \leqslant c_{2}
$$

and also

$$
c_{2}^{-1} \leqslant \frac{\left\|L_{\Omega}^{[n]} v\right\|}{\|v\| \prod_{0}^{n-1} \rho_{\mathscr{g}_{S} \Omega}}
$$

Proof. Let $v \notin C_{a, \Omega}$ (otherwise $L_{\Omega}^{[n]} \in C_{a, \mathscr{S} n_{\Omega}}$ ). Let $\zeta(\zeta \neq 0)$ be a complex number with smallest possible modulus such that $v+\zeta e_{\Omega} \in C_{a, \Omega}$. From Proposition A1 we have

$$
d_{\mathscr{S} n_{\Omega}}\left(L_{\Omega}^{[n]} v+\zeta L_{\Omega}^{[n]} e_{\Omega}, L_{\Omega}^{[n]} e_{\Omega}\right) \leqslant \mathcal{O}(1) \prod_{j=0}^{n-1} \theta_{\mathscr{Y}_{\Omega} \Omega}
$$

Let $u$ be the vector defined by

$$
u=\frac{L_{\Omega}^{[n]} v}{\zeta \prod_{0}^{n-1} \tilde{\rho}_{\mathscr{S} \Omega}}
$$

It follows from the hypothesis on $v$ and on $n$ that $u \notin C_{a, \mathscr{S}^{n} \Omega}$. We deduce from the above inequality

$$
\left\|\frac{u+e_{\mathscr{S n}_{\Omega}}}{1+\left\langle u \mid t_{\mathscr{S n}_{\Omega}}\right\rangle}-e_{\mathscr{S}^{n} \Omega}\right\| \leqslant \mathcal{O}(1) \prod_{j=0}^{n-1} \theta_{\mathscr{S}^{\prime} \Omega}
$$


or equivalently

$$
\left\|u-e_{\mathscr{S}^{n} \Omega}\left\langle u \mid t_{\mathscr{S}^{n} \Omega}\right\rangle\right\| \leqslant \mathscr{C}(1) \prod_{j=0}^{n-1} \theta_{\mathscr{S}^{\prime} \Omega}\left|1+\left\langle u \mid t_{\mathscr{P}^{n} \Omega}\right\rangle\right|
$$

We now deduce from the preceding lemma that

$$
\|u\| \leqslant c_{1}^{-1} \prod_{0}^{n-1} \theta_{\mathscr{P}_{\Omega} \Omega}(1+\|u\|)
$$

hence

$$
\|u\| \leqslant 2 c_{1}^{-1} \prod_{0}^{n-1} \theta_{\mathscr{S}_{/ \Omega} \Omega}
$$

if $n$ is larger than the smallest integer $n_{0}$ such that $\theta^{n_{0}} c_{1}^{-1} \leqslant 1 / 2$. For $n$ smaller than $n_{0}$ the first assertion of the lemma follows from the uniform bound on $\left\|L_{\Omega}\right\|$. For $n$ larger than $n_{0}$ it follows from the estimate on $\zeta$ (recall that $e_{\Omega} \in C_{a^{\prime}, \Omega}$ ):

$$
|\zeta| \leqslant \frac{1+a}{a-a^{\prime}}\|v\|
$$

If $v \in C_{a, \Omega}$, we have from Proposition A.1 and the relation between the norm and the Caratheodory distance

$$
\left\langle t_{\mathscr{P}^{n} \Omega} \mid L_{\Omega}^{[n]} v\right\rangle=\tilde{\rho}_{\mathscr{S}^{n-1} \Omega}\left\langle t_{\mathscr{S}^{n-1} \Omega} \mid L_{\Omega}^{[n-1]} v\right\rangle\left[1+\mathcal{O}(1) \theta^{n-1}\right]
$$

The result now follows by iterating this relation and using the definition of the cone.

We shall need later the projector on the vectors which do not have the same asymptotic growth rate as $e_{\Omega}$. The normal direction to this subspace is defined in the following lemma, where we suppose that $L_{\Omega}$ depends only on $p(\Omega)$ (since it is the case in the renormalization group analysis).

Lemma A.5. For $v$ in $\mathscr{E}$ the sequence of numbers $\alpha_{\Omega}^{(n)}(v)$ defined by

$$
\alpha_{\Omega}^{(n)}(v)=\frac{\left\langle t_{\mathscr{S n}_{\Omega}} \mid L_{\Omega}^{[n]} v\right\rangle}{\prod_{0}^{n-1} \tilde{\rho}_{\mathscr{S}_{\Omega} \Omega}}
$$

converges. The limit defines a family of bounded linear forms associated with a Hölder continuous family of vectors $\alpha_{\Omega}$ of $\mathscr{E}$. The direction of $\alpha_{\Omega}$ depends only on the components $\Omega$ of $\Omega$ with $j \geqslant 0$, and there is a positive constant $c_{4}$ such that for any $v$ in $\mathscr{E}$ and for any $\Omega \in p^{-1}(\omega)$, we have

$$
\left\|L_{\omega}^{[q]} v-\left\langle\alpha_{\Omega} \mid v\right\rangle L_{\omega}^{[q]} e_{\Omega}\right\| \leqslant c_{4} \theta^{q}\|v\| \prod_{0}^{q-1} \rho_{\mathscr{S} S \Omega}
$$


Proof. Assume first that $v$ belongs to $C_{a, \Omega}$. Then the convergence follows at once from Proposition A.1. It is easy to verify that $\alpha_{\Omega}$ defines an affine map on the cone $C_{a, \Omega}$. Since this cone is generating, we can extend $\alpha_{\Omega}$ to a continuous linear functional on $\mathscr{H}$. The convergence follows in the general case using Lemma A.4. The Hölder continuity follows as for $e_{\Omega}$ in Proposition A.2. For any vector $v$ and any integer $n$, we have

$$
L_{\Omega}^{[n]}\left(v-e_{\Omega} \alpha /\left\langle\alpha_{\Omega} \mid v\right\rangle\right) \notin C_{a, \varphi^{n} \Omega}
$$

(unless the left-hand side is zero). Indeed, if for some integer $n$ the above vector is in $C_{a, \mathscr{S}_{\Omega} \Omega}$, this will go on for any larger integer. Using Lemma A.4, we get a contradiction with the above argument. Let $\omega$ be an angle and let $\Omega \in p^{-1}(\omega)$. If $v$ is a vector in $\mathscr{E}$, we apply $L_{\omega}^{[q]}$ to each member of the decomposition

$$
v=\left\langle\alpha_{\Omega} \mid v\right\rangle e_{\Omega}+\left(v-\left\langle\alpha_{\Omega} \mid v\right\rangle e_{\Omega}\right)
$$

We deduce, using the above remark and Lemma A.4,

$$
\left\|L_{\omega}^{[q]} v-\left\langle\alpha_{\Omega} \mid v\right\rangle L_{\omega}^{[q]} e_{\Omega}\right\| \leqslant \mathscr{O}(1) \theta^{q}\|v\| \prod_{0}^{q-1} \rho_{\mathscr{S} J(\Omega)}
$$

In particular, this estimation leads to the following upper bound on $\left\|L_{\omega}^{[q]}\right\|$. For any $\Omega \in p^{-1}(\omega)$,

$$
\left\|L_{\omega}^{[q]}\right\| \leqslant \mathcal{O}(1) \prod_{0}^{q-1} \rho_{\mathscr{S} \Omega}
$$

Finally, if $\Omega$ and $\Omega^{\prime}$ differ only by their components of negative indices, it is easy to verify that

$$
\left\langle t_{\mathscr{S}^{n} \Omega} \mid L_{\Omega}^{[n]} v\right\rangle\left\langle\left\langle t_{\mathscr{S}^{n} \Omega^{\prime}} \mid L_{\Omega^{\prime}}^{[n]} v\right\rangle\right.
$$

converges to 1 . This implies that $\alpha_{\Omega}$ and $\alpha_{\Omega^{\prime}}$ are proportional.

Lemma A.6. There are positive constants $\varepsilon_{0}$ and $B$, and for any number $\varepsilon$ satisfying $0<\varepsilon<\varepsilon_{0}$, there is an integer $q(\varepsilon)$ such that if $q>q(\varepsilon)$, then

$$
l\left(\left\{\omega \mid \forall \Omega \in p^{-1}(\omega), \prod_{0}^{q-1} \rho_{\mathscr{S} S \Omega}>e^{q(\gamma+\varepsilon)}\right\}\right) \leqslant e^{-q B \varepsilon^{2}}
$$

Proof. It follows from well-known arguments (see, for example, ref. 3) that the thermodynamic limit

$$
\Phi(\beta)=\lim _{q \rightarrow \infty} q^{-1} \log \left(\int_{E} \prod_{0}^{q-1} \rho_{\mathscr{S}_{S} \Omega}^{\beta} d l(\Omega)\right)
$$


exists and defines a three-times-differentiable function $\Phi$ (see ref. 3 ). The result follows at once from the definition of $\gamma$ and the large-deviation theorem ${ }^{(12)}$ by noticing that there is a constant $C$ uniform in $q$ such that if $\Omega$ and $\Omega^{\prime}$ project on the same angle $\omega$, then uniformly in $q$ and in $\omega$, we have [since $d\left(\mathscr{S}^{j} \Omega, \mathscr{S}^{j} \Omega^{\prime}\right) \leqslant 2^{-j}$ ]

$$
\frac{\prod_{0}^{q-1} \rho_{\mathscr{S} \rho_{\Omega}}}{\prod_{0}^{q-1} \rho_{\mathscr{S} \rho_{\Omega^{\prime}}}} \leqslant C
$$

\section{APPENDIX B. THE INVARIANT FAMILY OF CONES IN THE E EXPANSION}

We now prove that the assumptions of Appendix A are satisfied in the case of the $\varepsilon$ expansion. We shall first recall some facts about this theory. It was proven in ref. 7 that for $\lambda$ positive and small enough, the Cvitanović equation

$$
\phi(\phi(\lambda x))=-\lambda \phi(x), \quad \phi(0)=1
$$

has a solution which is of the form $\phi(z)=\widetilde{\phi}\left(|x|^{1+\varepsilon}\right)$, where $\bar{\phi}$ is analytic in some (large) domain and $\varepsilon$ satisfies

$$
\varepsilon=\frac{-\lambda(1-\mathcal{O}(\lambda))}{1+\log \lambda}
$$

For simplicity we shall not indicate the $\lambda$ dependence of $\phi$ and $\tilde{\phi}$. As explained in ref. 7 , it is equivalent to considering the renormalization operator acting on $\tilde{\phi}$. As explained in Section 2, we consider (for $\varepsilon$ small enough) a fixed complex neighborhood $\mathscr{D}$ of the interval $[0,1]$ (for example, the set of points at distance 2), and sets of functions which are analytic in this neighborhood.

We can now check the hypotheses of Appendix A in the context of the $\varepsilon$ expansion. After some elementary computations, one gets the following estimate for the operator $L_{\omega}$ [acting on $h\left(|x|^{1+\varepsilon}\right)$ ]

$$
\begin{aligned}
L_{\omega} h(z)= & z\left\{h^{\prime}(0)+\cos \omega\left[h^{\prime}(1)+\operatorname{ch}(1) / \lambda\right]\right. \\
& \left.-2 i \lambda^{-1} \sin \omega\left[(1+\lambda) h(1)+\varepsilon h(1) / 2-\lambda h^{\prime}(1) / 2\right]\right\}+\lambda r(h) z
\end{aligned}
$$

where $h \rightarrow r(h)$ is an analytic map from a neighborhood $\mathscr{T}$ of $\tilde{\phi}$ in the set of functions analytic and bounded in $\mathscr{D}$. We now set for $\lambda$ small enough $a=1+1 / \log (-\log \lambda)$ and we define the invariant field of cones by

$$
C_{a, \omega}=\{h \in \mathscr{T}|\|h\| \leqslant a|\langle t \mid h\rangle \mid\}
$$


where $t$ is the function $t(z)=z /\|z\|$. We shall denote by $\delta_{\omega}$ the quantity

$$
\left\{[1+\cos \omega(1+\varepsilon / \lambda)]^{2}+4 \lambda^{-2} \sin ^{2} \omega(1+\lambda / 2+\varepsilon / 2)^{2}\right\}^{1 / 2}
$$

It is now easy to verify the hypotheses of Appendix A with $a^{\prime}=1+\lambda \log ^{2} \lambda$, $b=-\mathcal{O}(1) / \log \lambda$. Moreover, using [for $\left.\Omega \in p^{-1}(\omega)\right]$

$$
\rho_{\Omega}=\delta_{\omega}\left[1+\mathcal{O}(1) \lambda(\log \lambda)^{2}\right]
$$

one gets an estimate for the Lyapunov exponent $\gamma=-\log \lambda+\lambda+\mathcal{O}(1) \varepsilon$, and for the contraction factor $\theta_{\Omega} \leqslant \lambda^{1 / 2}|\log \lambda| / \delta_{\omega}$, from which it follows that $\theta_{\Omega} \rho_{\Omega}<e^{-\Gamma}$ for some positive (and large) number $\Gamma$.

\section{APPENDIX C. STABLE MANIFOLD}

In this section, we prove that for Lebesgue almost any angle, the renormalization iteration for a quasiperiodic noise has a (local) stable manifold. As explained in Section 3, this is not so relevant for the renormalization dynamics, because this stable manifold is of infinite codimension. In order words, in order to stay near the fixed point, one would have to satisfy infinitely many conditions (a phase transition of infinite order). As explained in Section 2, we need to make some assumptions on the dependence on the noise. As we have already explained, a continuous dependence is enough to ensure that the renormalization transformation is well defined. It is easy to show that the renormalization preserves this continuous dependence in a small neighborhood of the fixed point (in fact, it also preserves analyticity) because we are composing with an analytic function restricted to a smaller domain. More precisely, for any positive integer $p$, we denote by $\mathscr{A}_{p}$ the set of maps from $S^{1}$ to $\mathscr{B}_{1}$ such that their first $p$ derivatives has a square-integrable norm. Using the above remark, it is easy to verify that the renormalization operator maps a small enough neighborhood (in $\mathscr{A}_{1}$ ) of the constant function $\bar{\phi}$ into $\mathscr{A}_{1}$.

The technical difference with previous results on quasiproduct stable manifolds ${ }^{(20,22)}$ is precisely that our tangent map is not compact anymore. We shall use, however, the rather good control provided by the results of Appendix A. In some sense these results (hypotheses) overcome the problems created by large deviations. It would be interesting to deal with situations where this is not so. We shall use Mañés method ${ }^{(20)}$ suitably modified to take into account the fact that in our situation, the projection on the unstable manifold is not defined on the circle, but only in the extension $\{0,1\}^{\mathrm{Z}}$.

We now state the main result of this Appendix. 
Theorem C.1. For Lebesgue almost every angle $\omega$, there is a $C^{\infty}$ local stable manifold of the constant fixed point in $\mathscr{A}_{1}$.

Note that similar results hold in all Sobolev spaces.

Proof. We first recall briefly the method of proof used by Mañe. ${ }^{(20)}$ We first perform a translation so that the fixed point is now 0 . For each vector $v^{0}$ in $\mathscr{A}_{1}$ we construct a sequence $\left(v^{q}\right)_{q \in \mathrm{N}}$ in $\mathscr{A}_{1}$. We have used here upper indices because the lower indices are reserved for Fourier components. For a fixed (and small enough) projection of $v^{0}$ on the linear stable manifold $\mathscr{C}_{\omega}$, we shall determine by a fixed point equation the transverse part of $v^{0}$ so that the associated sequence $\left(v^{q}\right)_{q \in \mathbf{N}}$ is bounded (and in fact converges to 0 ).

We denote by $R_{\omega}$ the map obtained from $\mathfrak{R}_{\omega}$ after translating the fixed point at zero. It may be necessary to take some fixed iterate of this map to ensure that the constants appearing in the first part of Lemma A.4 are smaller than 1 . This may also require to have $\theta_{\Omega}$ slightly larger, but still smaller than one. We shall assume this is the case for only one iteration since the general argument requires only very elementary modifications. As observed in Section 2, $R_{\omega}$ is infinitely differentiable in $\mathscr{A}_{1}$ and we define the remainder operator $r$ by

$$
r(\omega, v)=R_{\omega}(v)-D R_{\omega}(0, v)
$$

We now define a Banach space of sequences $\mathscr{K}$. This is the space of sequences with elements in $\mathscr{A}_{1}$ equipped with the norm

$$
\left\|\left(v^{q}\right)_{q \in \mathbf{N}}\right\|=\sup _{q}\left\|v^{q}\right\| e^{-q c}
$$

where $c$ is a positive number to be chosen later. Let $\Pi_{\omega}^{l}$ be the projector with image in the contracted linear space $\mathscr{C}_{\omega}$ defined for $n \neq 0$ by

$$
\left(\Pi_{\omega}^{l} v\right)_{n}=v_{n}-\frac{\left\langle\alpha_{2^{i n \omega}} \mid v_{n}\right\rangle L_{n \omega}^{[l]} \alpha_{n \omega}}{\left\langle\alpha_{2^{i n \omega}} \mid L_{n \omega}^{[l]} \alpha_{n \omega}\right\rangle}
$$

and the projection on the stable eigenspace of the usual linearized renormalization for $n=0$. In the above formula, $\alpha_{\omega}$ is not well defined. However, from Lemma A.5 it follows that the direction of $\alpha$ depends only on $\omega$, and since the above formula is projective in $\alpha$, there is no ambiguity. We also define $\widetilde{\Pi}_{\omega}^{l} v=v-\Pi_{\omega}^{l} v$. Note that it follows easily from Lemma A.5 that the vector

$$
\frac{L_{\omega}^{[l]} \alpha_{\omega}\left\|\alpha_{\omega}\right\|}{\left\langle\alpha_{2^{l} \omega} \mid L_{\omega}^{[l]} \alpha_{\omega}\right\rangle}
$$


has a norm which is uniformly bounded. It then follows that the above projectors have a norm uniformly bounded in $\mathscr{A}_{\infty}$ (with respect to $l$ and to $\omega$ ).

From now on, we shall assume that $\sup _{\Omega} \theta_{\Omega} \rho_{\Omega}<e^{-\Gamma}$, where $\Gamma$ is a positive number. We recall that this inequality is true at least in $\varepsilon$ expansion (see Appendix B). It now follows easily from Lemma A.4 that

$$
\left\|\mathscr{L}_{2}^{[q]} \Pi_{\omega}^{l} \Pi_{\omega}^{l} v\right\| \leqslant e^{-q \Gamma}\|v\|
$$

where again the above inequality may be true only for some (fixed) iterate and a slightly larger $\theta_{\Omega}$.

Let $\psi$ be a positive number to be chosen later. We define a family of maps $T_{\omega, w^{0}}$, where $w^{0} \in \mathscr{C}_{\omega}$, by

$$
\begin{aligned}
T_{\omega, w^{0}}(\underline{v})^{q}= & \mathscr{L}_{\omega}^{[q]} \Pi_{\omega}^{0} v^{0}+\sum_{0}^{q-1} \mathscr{L}_{2^{\prime+1}{ }_{\omega}}^{[q-1]} \Pi_{\omega}^{j+1} r\left(2^{j} \omega, v^{j}\right) \\
& +\sum_{q}^{\infty}\left(\mathscr{L}_{2^{q} \omega}^{[j-q+1]} \tilde{\Pi}_{\omega}^{q}\right)^{-1} \tilde{\Pi}_{\omega}^{j+1} r\left(2^{j} \omega, v^{j}\right)
\end{aligned}
$$

for $q \neq 0$, and

$$
T_{\omega, \mathcal{W}^{0}}(\underline{v})^{0}=w^{0}-\sum_{1}^{\infty}\left(\mathscr{L}_{\omega}^{[j+1]} \tilde{\Pi}_{\omega}^{0}\right)^{-1} \tilde{\Pi}_{\omega}^{j+1} r\left(2^{j} \omega, v^{j}\right)
$$

if $\left\|w^{0}\right\|$ and $\|\underline{v}\|$ are smaller than $\psi$.

It is easy to verify that for a small enough $\psi$ and a fixed $\omega, T_{\omega, w^{0}}$ is defined and $C^{\infty}$ on some neighborhood of 0 in $\mathscr{K}$ and the image is also in $\mathscr{K}$ if $c$ is small enough $(c<\Gamma)$. It now follows as in ref. 20 that a necessary and sufficient condition for a vector $v^{0}$ of norm less than $\psi$ and projection $w^{0}=\Pi_{\omega}^{0} v^{0}$ on $\mathscr{C}_{\omega}$ to be on the stable manifold at the angle $\omega$ is that there exists a sequence $\underline{v}$ in $\mathscr{K}$ which is a fixed point of $T_{\omega, w^{0}}$. The existence (and uniqueness) of such a sequence for a $w^{0}$ of small enough norm follows from the implicit function theorem ${ }^{(10)}$ as in ref. 20 . The regularity of the manifold follows similarly.

\section{ACKNOWLEDGMENTS}

P.C. would like to thank J. Lebowitz and the Mathematics Department of Rutgers University, where part of this work was done. This work was partially supported by NSF grant DMR 86-12369. 


\section{REFERENCES}

1. F. Argoul and A. Arnéodo, J. Mech. Theor. Appl. (Special volume) 1984:241 (1984); J. Phys. Lett. (Paris) 46:L901 (1985).

2. F. Argoul, A. Arnéodo, P. Collet, and A. Lesne, Europhys. Lett. 3:643 (1987).

3. R. Bowen, Lecture Notes in Mathematics, Vol. 470 (Springer, New York, 1975).

4. T. Bohr and D. Rand, Physica 25D:387 (1986).

5. P. Collet and J.-P. Eckmann, Iterated Maps of the Interval As Dynamical Systems (Birkhauser, Boston, 1980).

6. P. Collet, J.-P. Eckmann, and H. Koch, J. Stat. Phys. 25:1 (1981).

7. P. Collet, J.-P. Eckmann, and O. E. Lanford, Commun. Math. Phys. 76:211 (1980).

8. J. Crutchfield and B. Huberman, Phys. Lett. A 77:405 (1980).

9. J. Crutchfield, M. Nauenberg, and J. Rudnick, Phys. Rev. Lett. 46:933 (1981).

10. J. Dieudonné, Éléments d'analyse (Gauthier Villars, Paris, 1969).

11. J. Dixmier, Les algèbres d'opérateurs dans l'espace Hilbertien (Gauthier Villars, Paris, 1957).

12. R. Ellis, Entropy, Large Deviations and Statistical Mechanics (Springer, New York, 1985).

13. H. Epstein, in Nonlinear Evolution and Chaotic Phenomena, G. Gallavotti and P. Zweifel, eds. (Plenum Press, New York, London, 1988).

14. C. Earl and R. Hamilton, Proc. Symp. Pure Math. XVI (1970).

15. D. Fiel, J. Phys. A Math. Gen. 20:3209-3217 (1987).

16. P. Ferrero and B. Schmitt, Prob. Theory Rel. Fields 79:227 (1988).

17. J. M. Gambaudo, J. Loss, and C. Tresser, Preprint, University of Nice (1987).

18. M. Herman, Comment. Math. Helv. 58:453 (1983).

19. O. E. Lanford and R. Llave, to appear.

20. R. Mañé, in Lecture Notes in Mathematics, Vol. 1007 (Springer, New York, 1983).

21. P. Martin, B. Schraiman, and E. Wayne, Phys. Rev. Lett. 46:935 (1981).

22. D. Ruelle, Ann. Math. 115:243 (1982).

23. E. Vul, K. Khanin, and Y. Sinai, Russ. Math. Surv. 39:1 (1984). 